\title{
Prospective Financial Assessment Based on Real Options in Small and Medium-Sized Company
}

\author{
Bartłomiej Nita*, Piotr Oleksyk*, Jerzy Korczak**, Helena Dudycz* \\ *Wrocław University of Economics, Wroclaw, Poland \\ **International University of Logistics and Transport, Wrocław, Poland
}

Email: \{bartlomiej.nita, piotr.oleksyk, jerzy.korczak, helena.dudycz\}@ue.wroc.pl

\begin{abstract}
The article presents a prototype of an intelligent Early Warning System based on real option approach to prospective financial assessment of Small and Medium-Sized Enterprises (SME). The described approach constitutes a continuation of development of the intelligent cockpit for managers (InKoM project), the main objective of which was to facilitate financial analysis and evaluation of economic status of a company. The current project is related to the design of smart evaluation of critical financial situations of SME using real options, domain ontology, and AI methods. The content of the knowledge is focused on essential financial concepts and relationships connected with risk assessment, taking into consideration internal and external economic and financial information. A case study based on the real option has been carried out on financial data extracted from financial information system.
\end{abstract}

\section{INTRODUCTION}

$\mathrm{T}$ ODAY, innovative methods combined with advanced financial analysis tools are required to correctly assess critical economic standings of Small and Medium-Sized Enterprises. The main stumbling block and difficulty is that managers of SMEs often do not possess solid background knowledge in financial analysis and new available IT solutions, in particular Decision Support Systems. The problem is often caused by lack of the knowledge required to correctly interpret economic indicators. This knowledge may be improved using real option approach to investment appraisal in SME.

In general, an enterprise works better on the competitive space if it tries to identify development opportunities and threats of disruption a company's leading activity. This requires implementation of prospective financial assessment in SME. Most of SME managers are not skilled enough to understand and respond to threats coming from the business environment. Real options have been applied in practice to solve such issues as just described. In addition, they are treated as the risk management instruments used to assess financial risk of high-risk development projects as well as to influence the company's ability to continue as a going concern in the future [1-2]

Taking into consideration all managerial requests and the complexity of business problems, solutions are needed to integrate managerial knowledge and computational methods so as to support intelligent analysis and decision making [3].
The aim of the paper is to present the of a prototype based on the real option approach that integrates financial knowledge, predictive models, and business reasoning to support financial assessment in Early Warning Systems. The term "real option" can be defined using the analogy to the financial option. Real option therefore means the right of its holder to buy or sell some underlying assets (basic instrument, which is usually an investment project) in specified sizes, at a fixed price and at a given time [4, p. 172]. Generally, it can be said that the real option is the right to modify an investment project in an enterprise [5, p. 269].

Thus, we demonstrate how to employ real option approach in Early Warning Systems to support financial assessment in SME with the aim of avoiding bankruptcy. In our project, it is assumed that financial knowledge is formally defined by the domain ontology. The essential part of the work is to develop a smart solution facilitating automated analysis of information available in financial databases and external data.

The paper has been structured as follows. The first part in a critical way introduces the application of Early Warning Systems in the context of financial assessment. In the succeeding section, the concept of application of real option for the purposes of investment appraisal is discussed. In section three, knowledge conceptualization and reasoning are elaborated, with proposal of an appropriate ontology. Next we present a case study explaining the prototype that refers to prospective financial assessment based on real option approach. Finally, in the last section, some conclusions are drawn and the future directions of the project discussed.

\section{CRITICAL ANALYSIS OF EARLY WARNING SYSTEMS IN THE CONTEXT OF FINANCIAL ANALYSIS}

Early warning is a process which allows an organization to consistently anticipate and address competitive threats. As far back as early seventies, managers of firms had started thinking about methods that would allow for early identification of opportunities and threats present in their business environment. It led to the emergence of Early Warning Systems, which were to as early as possible forewarn of approaching threats and opportunities and explore their weak signals. Many methods have been developed to analyze SME performance aimed at creating the Early Warning 
System [6]. Unfortunately, they are more often based on past data, and this at present is simply not enough. The essential requirement for SME to survive in a competitive market is development of mechanisms allowing for generation of revenues from core operations in the future. In planning future activities, company's managers emphasize the need to maintain existing customers. If this is not possible, attempts are made to search for new customers. It is also necessary to analyze competitive actions, which in the near future could lead to a significant decrease in market share.

One of the main weaknesses of existing Early Warning Systems is the lack of a formal representation of the knowledge and analytical models that take into consideration both internal and external information. Managers using simple Early Warning Systems receive various alerts, but they don't know which problems should be addressed first. Moreover, these systems do not indicate for managers which suggestions are to be implemented, hence managers have to rely solely on their managerial intuition. It is therefore necessary to extend the EWS functionality.

The proposed prototype is focused on prospective information as well as value embedded in real options. Financial forecasts serve as the basis for the remedial actions that take into account contingent factors. Such activities are focused on searching for value hidden in real options, so as to take advantage of opportunities that may emerge in the future. This is not possible without extending the EWS with regard to external information. This kind of external information is not formalized, thus this extension is another challenge. Moreover, external information may be supplemented with data processing algorithms based on management accounting and finance learning tools.

\section{REAL OPTIONS IN ASSESSING INVESTMENTS}

Standard approach to investment appraisal is based on discounted cash flow methodology, in particular NPV (Net Present Value) analysis. This approach is currently insufficient mainly due to the high volatility of external factors affecting a company [7-8]. The commonly used net present value criterion is currently considered as static mainly because it is calculated at a given moment and does not anticipate changes that may occur in the future. As a result, the NPV criterion does not take into account the opportunity to react to new circumstances, such as [9]:

- an unexpected collapse of the market, which leads to a reduction in the business size,

- significant changes in prices, which may have a significant impact on the profitability of the project,

- an exceptionally favorable situation that allows for expanding the scope of activities.

Taking into account of the limitations of NPV criterion, address suggest to apply the concept of real options ${ }^{1}$. Techniques based on the net present value are still necessary and valuable, hence they should not be underestimated in

1 The term „real option” was initially used in 1977 by S.C. Myers from Massachusetts Institute of Technology [10]. This concept was further developed by A.K. Dixit and R.S. Pindyck [11]. any case. However, real options allow for a deeper analysis of the investment appraisal issue and somehow expand the traditional methods due to the identification of various investment possibilities embedded in the investment projects. Jahanshahi et al. [12] argues the role that real options can play in SME to increase market orientation and organizational learning, consequently providing a firm with the ability to both attain and sustain competitive advantage, particularly in a volatile environment.

The value of this flexibility is reflected in the option price (option premium); it increases if the probability of receiving new information increases and ability to risk bearing increases. The value of this flexibility is the difference between the value of the investment project with the right of managers to modify the project embedded and the value of the project in the absence of managerial discretionary to modify project. This relationship can be described as follows [see: 13]:

$$
S-N P V=N P V+O V
$$

where $S-N P V$ - a strategic net present value,

$N P V$ - a standard (static, passive, direct) net present value $O V-$ an option value.

The lack of flexibility is especially the main factor preventing managers from taking risk. Power and Reid [14] test empirically whether real options logic applies to small firms implementing significant changes (e.g. in technology). Their research findings imply that strategic flexibility in investment decisions is necessary for good long-run performance of small companies.

The valuation of real options is a difficult task and very often impossible to be carried out by manager of SME. It should be noted that value of real options is closely linked with high risk. A manager without advanced financial knowledge can increase the level of risk associated with running a business. Thus, it is necessary to build a prototype that will guide the manager through all the risks associated with the investment project taking into account contingency factors. The prototype also indicates additional opportunities which result from the company's environment. It is also required to create a smart analytical tool that processes signals coming from the environment and integrates them with the real option pricing module.

\section{KNOWLEDGE CONCEPTUALIZATION AND REASONING}

Implementation of any development project should be preceded by a multi-faceted analysis confirming its profitability. The objective of analytical activities, mainly based on external sources, is focused on confirmation of the necessity of unconditional implementation of changes in the enterprise. After obtaining external information, it is necessary to integrate them with data describing an entity's potential to implement new projects and solutions.

The prototype includes extended analytical methods. This method is aimed at integrating data from internal reporting with external information. In the prototype, it is assumed that business knowledge is formally described using 


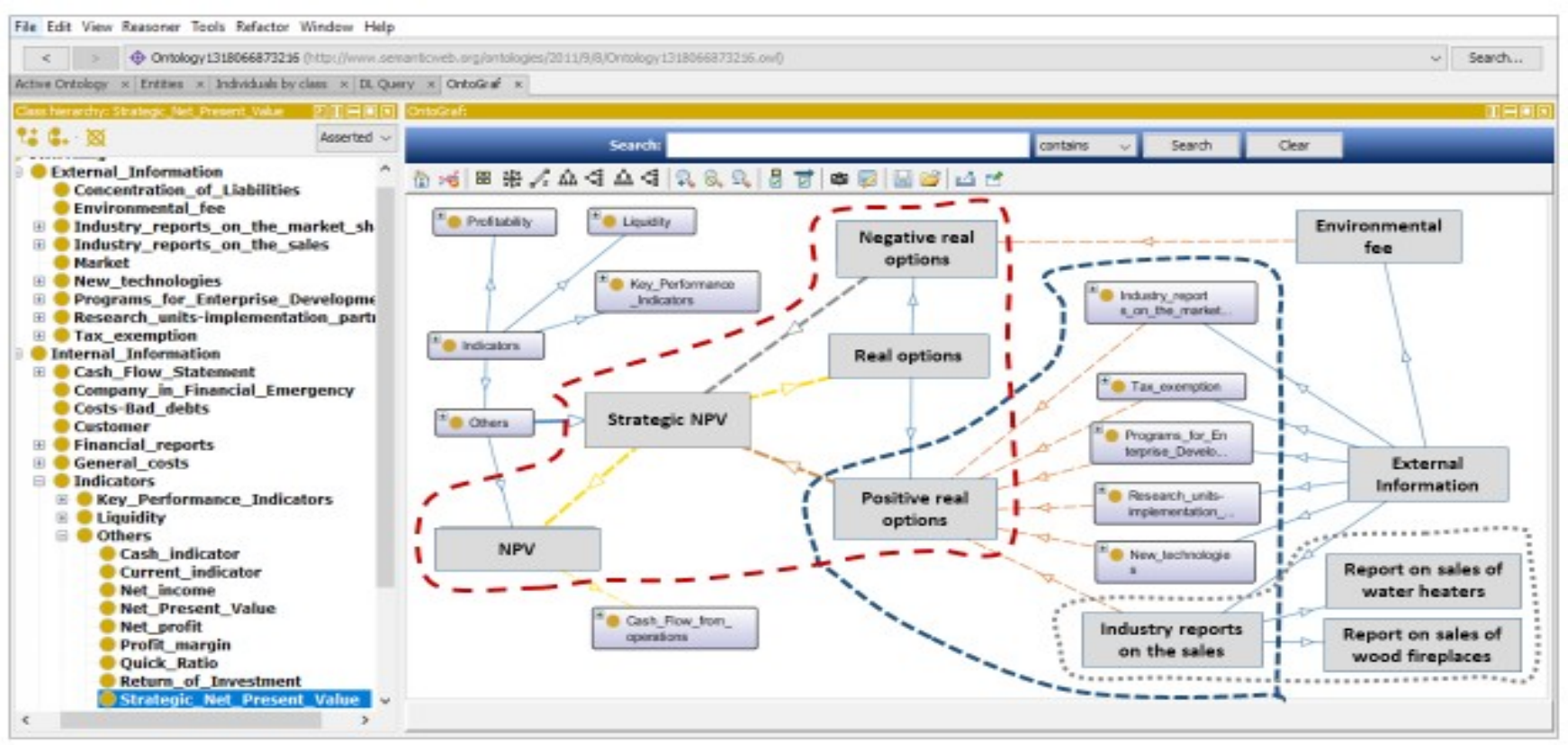

Fig. 1. An example of visualization of a semantic network of Strategic NPV Source: own elaboration using Protégé editor.

ontology ${ }^{2}$, which is one of commonly used method of representing knowledge in the information systems. The ontology includes internal information and external information essential for the functioning of the company. A manager can browse hierarchy of concepts, relationships, and annotations. In addition, post conditions such as positive and negative effects of decision process execution can be defined.

The ontology has been encoded using the Protégé platform (http://protege.stanford.edu/). It is important to note that the given ontology describes only static structures, namely the financial concepts and their relationships and external information having influence on the functioning of an enterprise (especially for SME). The ontology presented in Fig. 1 shows a few concepts related to the analysis of the strategic NPV.

Figure 1 illustrates a sample visualization of external and internal information focused on the issue of the strategic NPV. There are two panels on the screenshot. The panel to the left shows taxonomic relations, while the one to the right allows for visualization of taxonomic and semantic relations between defined topics (semantic network visualization). There are two types of lines between topics: (1) the solid line represents a relation subclass-of and (2) the dashed line represents the experts' defined relationships (for example: depends on) on the figure.

Figure 1 presents topics important topics in rectangles for the analysis of the strategic NPV. The presented part of the ontology shows that Strategic NPV depends on standard $N P V$ and Real options, which contain Positive real options and Negative real options. Positive real options increase strategic NPV, while negative real options decrease Strategic NPV. Instances of positive and negative real options are External information (for example: Industry reports on the sales, Environmental fee). This part of ontology shows to the manager, that if he wants to calculate Strategic NPV, he should estimate Real options. The manager can see that he should analyze External information affecting the calculation of the positive and negative values of real options. The manager can add, modify as well as retrieve topics related to the problem at hand.

Our proposal to extend the functionality of the system is based on introduction of financial ontology, containing internal and external variables related to real options, as described in Fig. 2. The system processes selected information from financial reports and business environment, subsequently forecasting a company's economic and financial situation. In a situation of a negative forecast, in addition to warning messages, it indicates the possibility of using the real options that would allow a manager to exit a critical situation. The financial ontology not only helps identify the concepts and relationships between them, but it also helps in the interpretation of the current and future situations of the company.

In order to explain the operation of the system, let's describe the vector of the input information as follows:

$$
\left[\left(f_{l}, \ldots, f_{k}\right),\left(e_{1}, \ldots, e_{l}\right),\left(r_{1}, \ldots r_{m}\right),\left(\delta_{l}, \ldots \delta_{n}\right)\right]
$$

where $f_{l}, \ldots, f_{k}$ denote information from financial reports, $e_{l}, \ldots, e_{l}$ information from the business environment, $r_{l}, \ldots r_{m}$ information about real options, $\delta_{1}, \ldots \delta_{n}$ are estimates of future changes of variable values.

2 Ontology "is an explicit specification of a conceptualization" $[15$, p. 907]. 


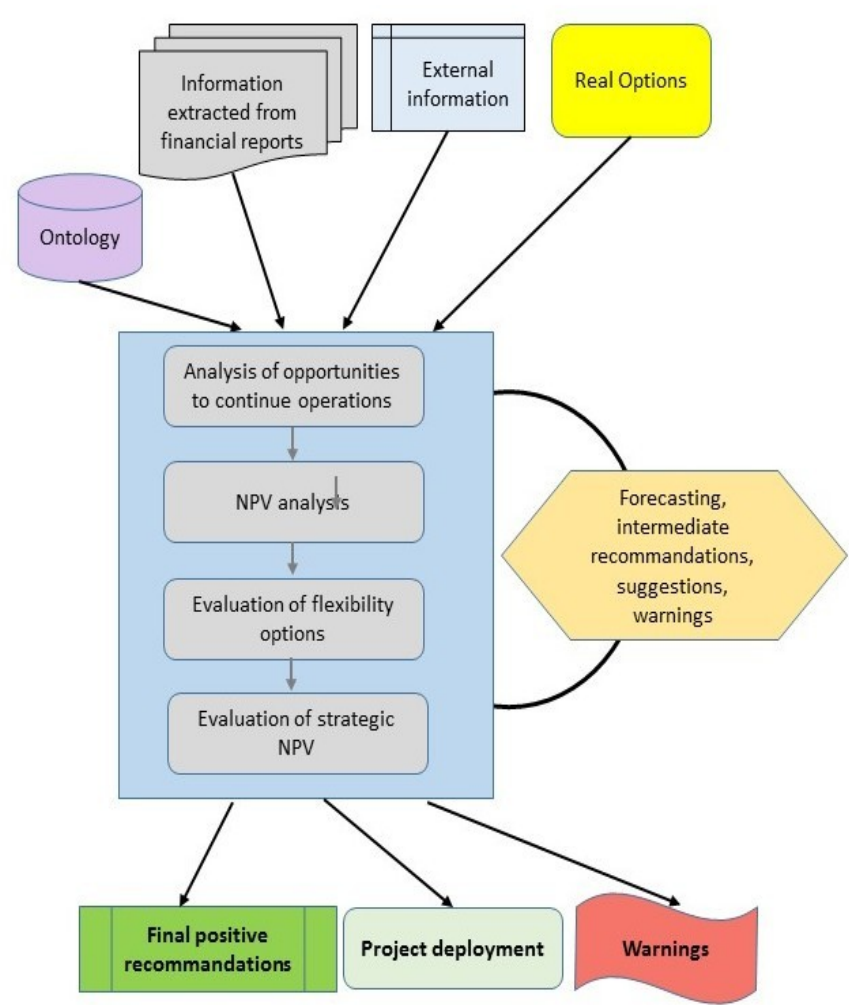

Fig. 2. Functional schema of smart EWS

This information is interpreted by the decision rules described in the ontology, for example:

\section{if $f_{i}{ }^{\circledR}$ threshold $f$}

then message $f$-warning else message f-positive

where $₫$ denotes a specific relationship between values of $f_{i}$ and the threshold.

Depending on the values and number of thresholds, the messages can be more or less varied. If we left the output messages in this form, then the logic of our system would not be different from the classic EWS.

We have introduced several new solutions in the project. The first is the transformation of information qualification into the values of multivalued logic. An example of a transformation with regard to NPV is shown in Fig. 3.

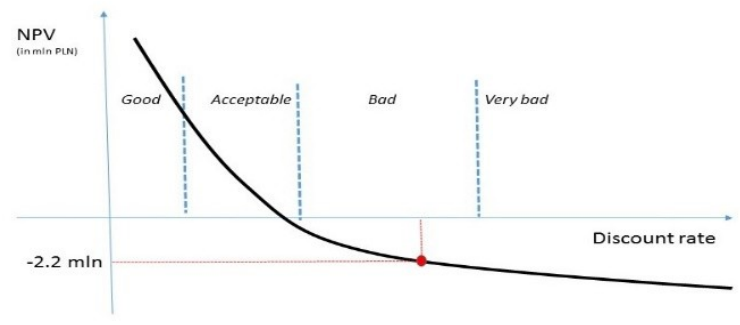

Fig. 3. Example of values transformation

In order to assess the overall situation of the company, these values (Very bad, Bad, Acceptable, and Good) can be transformed into numeric values, for example $(2,3,4,5)$, and use in computational models. Additionally, a manager can give weights to variables in the range prototype $[0,1]$, where 0 is a negligible variable, and 1 is a very important variable. In the prototype, the weights of variables may be taken by default or defined by a manager.

With these assumptions, assessment of the company $S$ situation can be computed in two ways: as an arithmetic average or as a geometric mean:

$$
\begin{aligned}
& S=\Sigma\left(f_{i}^{*} w_{i}+e_{j},{ }^{*} w_{j}+\left\{r_{k}{ }^{*} w_{k}+\delta_{l} * w_{l}\right\}\right) / m \\
& \text { or } \\
& S=\sqrt{ } f_{i}{ }^{*} w_{i} * e_{i},{ }^{*} w_{j} *\left\{r_{k}{ }^{*} w_{k} * \delta_{l} * w_{l}\right\} / m .
\end{aligned}
$$

Note that the evaluation is performed twice, first without taking into account variables related to real options and estimating the increase in values resulting from accepting real options (expression $\left\{r_{k}{ }^{*} w_{k}+\delta_{l}{ }^{*} w_{l}\right\}$, and the second time after accepting the real options. Interpretation of the assessment and its justification are prepared using financial ontology and data from an enterprise's information system.

The design of an inference process illustrating this concept will be discussed in the next section.

\section{CASE STUdY}

To illustrate the need for real option valuation, we present the case of a project that would be rejected on the basis of traditional analytical methods. Based on valuation of flexible option to expand, we have shown that to avoid going bankrupt, the management should choose_to implement the project.

Assumptions of the case study:

- managers of a manufacturing company producing water heaters and wood fireplaces, while preparing sales forecasts, identify a significant problem with the company's ability to continue its operations,

- managers, based on their expertise and experience, foresee that if they decide to abandon development projects, the company will lose the ability to continue its operations within 5-7 years,

- when planning innovations in the enterprise, a new design of a fully ecological cogeneration fireplace meeting the most stringent ecological standards has been developed,

- the forecasted product cost suggests high selling price that does not allow for launching the project,

- it is necessary to implement changes in production technology, that would make it possible to reduce costs and offer a lower price of the new product, however, NPV analysis indicates that the project would still be unprofitable.

Due to the limited size of the article, it is not possible to indicate a detailed valuation of the real option, and thus strategic value of NPV. Such activities require a large number of calculations, calibration of input parameters, and adoption of discretionary assumptions for the valuation of future benefits. The prepared EWS prototype allows the manager to assign any rank to each source of information. 
However, less experienced managers can use the hint embedded in the prototype, which suggests default solutions.

The prototype of the system presented in Fig. 2 contains next analytical steps. The ontology built into the prototype (Fig. 1) explains to the manager the basic concepts and problems associated with the sales profitability. The ontology also presents the knowledge that combines the profitability issue with the investment project appraisal. The manager receives information from the system that it is not possible to conduct development activities. The knowledge contained in ontology explains to the manager the essence of valuation of the flexibility option. The system presents a set of fundamental information needed to evaluate the flexibility option, but the information should be verified by the manager. The manager may or may not expand this information base based on his own expertise. In the analyzed company, the situation allows for initiating preparatory activities to launch the project.

Improvement of the financial situation should be the dominant objective of any manager. It is very difficult for the manager to determine the right moment to implement an investment. Contextual analysis of the impact of real options on a given project is very often beyond the scope of most SME managers. The SME manager is not in a position to take into consideration all the aspects of the development project on his own. It is often necessary to hire a consultant, which is an additional cost. Therefore, creation of a prototype that makes it possible to handle prospective analysis seems indispensable.

\section{CONCLUSION AND FUTURE WORKS}

The main objective of the paper was to present foundations of a prototype based on real option approach that incorporates financial knowledge, predictive models, and business reasoning to support financial assessment in Early Warning Systems. The implemented prototype contains unique methods of prospective analysis used to assess profitability of an investment project. The novelty of the approach consists in applying real options embedded in the Early Warning System. The example is based on real data extracted from a small company. Risk of bankruptcy could be avoided by making decisions based on intelligent indepth analysis of external information combined with the analysis of financial situation that allows for implementation of corrective solutions.

From a financial perspective, the presented case study supports the conclusion that the decision to undertake any investment cannot be based solely on estimation of standard NPV. It also requires analysis of various external factors determining decision making process. Managers of SMEs may take advantage of the proposed system that integrates financial knowledge and predictive models. Therefore, the system provides knowledge not only on the required internal information from various reports, but also from external information (which are weak signals). The proposed ontology seems to be a promising extension to Early Warning Systems. It not only improves the quality of analysis, but also enhances managerial ability to better understand relations between financial data (internal information) and various factors affecting development of the SMEs (external information).

Further work should be focused on a global process-oriented approach to financial assessment. This will not be possible without large databases of real case studies and use of knowledge possessed by experienced managers and financial analysts. For a company, the multidisciplinary approach to develop the prospective analysis in the Early Warning System could contribute to attainment of a competitive advantage and to increase its financial stability.

\section{REFERENCES}

[1] M. Benaroch, Y. Lichtenstein and K. Robinson, Karl. 2006. "Real Options in Information Technology Risk Management: An Empirical Validation of Risk-Option Relationships," MIS Quarterly, 2006, vol. 30 (40), pp. 827-864

[2] G. Favato and R. Vecchiato, "Embedding real options in scenario planning: A new methodological approach, Technological Forecasting and Social Change, Elsevier, 2017, vol. 124(C), pp. 135-149, DOI: 10.1016/j.techfore.2016.05.016

[3] J. Korczak, H. Dudycz, B. Nita, P. Oleksyk and A. Kaźmierczak, “Attempt to extend knowledge of Decision Support Systems for small and medium-sized enterprises", in: Proc. of the 2016 Federated Conference on Computer Science and Information Systems, M. Ganzha, L. Maciaszek, M. Paprzycki, Eds., Annals of Computer Science and Information Systems, vol. 8, 2016, pp. 1263-1271, DOI:10.15439/2016F181

[4] B. Nita, „Metody wyceny i ksztaltowania wartości przedsiębiorstwa” [Methods of Corporate Valuation and Value-Based Management], PWE, Warszawa 2007.

[5] R. A Brealey and S. C. Myers, Principles of Corporate Finance, McGraw Hill, 2003.

[6] A.S. Koyuncugil and N. Ozgulbas (Eds.), Surveillance Technologies and Early Warning Systems: Data Mining Applications for Risk Detection, IGI Global, 2009

[7] T. Adelaja, Capital Budgeting: Capital Investment Decision Paperback, CreateSpace Independent Publishing Platform, 2016

[8] U. Götze, D. Northcott and P. Schuster, Investment Appraisal: Methods and Models, Springer, 2015

[9] A. Damodaran, "The Promise of Real Option", in: J. M. Stern, D. H. Chew, Eds., The Revolution in Corporate Finance, Blackwell Publishing, 2003.

[10] S. C. Myers, "Determinants of Capital Borrowing", Journal of Financial Economics, 1977, vol. 5., pp. 147-175

[11] A. Dixit and R.S. Pindyck, Investment under Uncertainty, Princeton University Press. Princeton, New Jersey 1994

[12] A. Jahanshahi, K. Nawaser, N. Eizi and M. Etemadi, "The Role of Real Options Thinking in Achieving Sustainable Competitive Advantage for SMEs", Global Business \& Organizational Excellence, November 2015 , vol. 35 , no. 1 , pp. 35-44

[13] L. Trigeorgis, Real Options. Managerial Flexibility and Strategy in Resource Allocation, The MIT Press, Cambridge 1998

[14] B. Power and G. Reid, "Organisational change and performance in long-lived small firms: a real options approach", European Journal Of Finance, September 2013, vol. 19, no. 7/8, pp. 791-809

[15] T. R. Gruber, Toward Principles for the Design of Ontologies Used for Knowledge Sharing, Technical Report KSL, Knowledge Systems Laboratory, Stanford University, 1993, http://tomgruber.org/writing/ontodesign.pdf 